\title{
Submandibular Swelling, Pulsations and Otalgia Due to Sublingual Gland and Blood Vessels Herniation Through Mylohyoid Boutonnière: Case Report
}

\begin{abstract}
SUMMARY
Background/Aim: Mylohyoid boutonnière is a common anatomical variant with extremely rare clinical manifestations. We report two cases of symptomatic unilateral mylohyoid boutonnière with sublingual gland herniation. Case report: The first was a 34-year-old female with a two months history of a left submandibular swelling, intermittent pulsations, and otalgia. The second 67-year-old female presented with a left submandibular pain six months after total thyroidectomy due to papillary carcinoma. After detailed clinical and radiological evaluation by ultrasonography (US) and computed tomography (CT), the mylohyoid gap with sublingual gland herniation was diagnosed in both patients. The dynamic US with a tongue pressed down on the mouth floor revealed blood vessel compression by a herniated sublingual gland in the first patient. Conclusions: This is the first case of a mylohyoid boutonnière related intermittent vascular compression. Dynamic US and CT may help to differentiate intermittent and persistent sublingual gland herniation through the mylohyoid boutonnière from other pathological lesions in the submandibular region.
\end{abstract}

Key words: Anatomic Variation, Mylohyoid Muscle, Sublingual Gland, Ultrasonography, Computed Tomography
Aleksa Janović, Djurdja Bracanović, Svetlana Antić, Biljana Marković-Vasiljković

Department of Diagnostic Radiology, Faculty of Dental Medicine, University of Belgrade, Belgrade, Serbia

\section{CASE REPORT (CR)}

Balk J Dent Med, 2022;58-62

\section{Introduction}

Mylohyoid muscle is a pared flat suprahyoid muscle that attaches along the mylohyoid line on the mandibular body superiorly, and the body of the hyoid bone inferiorly ${ }^{1}$. Muscles from each side are connected via the median fibrous raphe thus forming the muscular floor of the oral cavity ${ }^{1}$. It has been thought that mylohyoid muscles separate the submandibular and the sublingual space completely and that these two spaces communicate only behind the posterior border of the mylohyoid muscle. However, Forget ${ }^{2}$ was the first who described the presence of a gap in the mylohyoid muscle of a cadaver. Soon after him, Delens ${ }^{3}$ reported a similar gap in a cadaver with the sublingual gland herniating partially through it. Due to the similarity with a buttonhole, the author named this gap as „,boutonnière musculaire“ ${ }^{\text {‘3 }}$.
Systemic anatomical and radiological studies revealed that the mylohyoid boutonnière is a relatively common finding in asymptomatic individuals with prevalence up to $58 \% 4-10$. It is round or fissure-like opening approximately $15 \mathrm{~mm}$ in size, most commonly located in the anterior third of the mylohyoid muscle adjacent to the mandibular body $4,5,7,10$. Mylohyoid boutonnière may be found bilaterally in almost one-third of a population ${ }^{5-7,9}$. Fat, communicating vessels between the sublingual and submandibular artery, and a part of the sublingual gland was described within the mylohyoid boutonnière in the descending order.

Even though the presence of a mylohyoid boutonnière may potentially facilitate spreading of pathological processes from sublingual to the submandibular space and vice versa, authors were not able to perceive any other clinical significance of this anatomical variation. In the current paper, we presented 
two cases of symptomatic herniation of the sublingual gland and blood vessels through the mylohyoid boutonnière.

\section{Case Report}

\section{Case Report I}

The first patient was a 34-year-old non-smoker female who was referred to the Clinic of Maxillofacial Surgery with two months-long history of a left submandibular swelling. The swelling was accompanied by intermittent pulsations and moderate pain that irradiates to the left ear. The patient also complained of periodic swallowing difficulties and weight loss of approximately 6 kilograms during the last year. No other significant medical or family history was reported. On physical examination, there was a round firm and painful palpable mass in the left submandibular region, approximately $1 \mathrm{~cm}$ in size. The mass was relatively freely movable without changes in the overlying skin. No other abnormalities were seen. Routine laboratory blood analyses (leukocyte formula, C-reactive protein) were within normal ranges. Lymphadenopathy, salivary gland tumor, and developmental cyst were the main considerations.

Ultrasound (US) examination (Philips Affinity 70G, $12 \mathrm{MHz}$ linear probe) revealed a well-defined homogenous iso to the hyperechoic formation in the left sublingual and submandibular space with the hourglasslike shape (Figure 1). Submandibular glands and lymph nodes appeared normal. Given that this finding resembled the plunging ranula, a computed tomography (CT) examination was recommended in order to evaluate deep soft-tissue structures of the mouth floor.

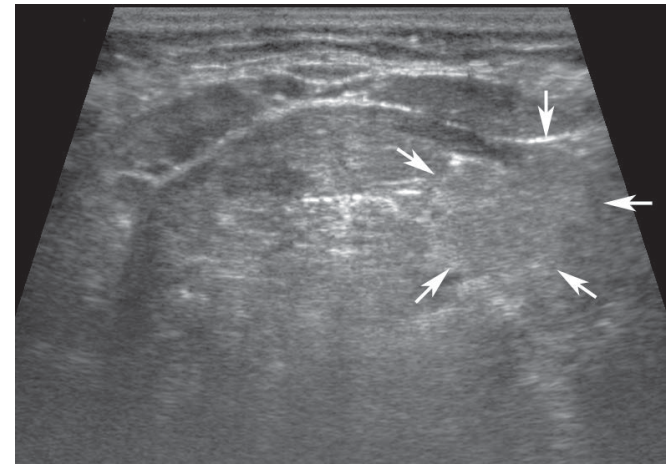

Figure 1. Axial US image of the submental region of the neck $(12 \mathrm{MHz}$ linear probe). Note the hourglass-like shaped formation in the left sublingual space protruding into the submandibular space (arrows)

Contrast-enhanced CT examination (64-slice Philips Ingenuity CT, Philips Medical systems, Cleveland, USA) showed left-sided hypertrophy of the sublingual salivary gland protruding through the mylohyoid muscle gap into the submandibular space (Figure 2a,b). The gap was $5 \mathrm{~mm}$ in the anteroposterior diameter and located in the anterior third of the muscle, near the mandibular body. The left sublingual gland was homogenously enhanced without detectable focal pathological changes. A smaller rightsided mylohyoid defect was also depicted, but without the sublingual gland herniation (Figure 2b). There were also blood vessels passing through the mylohyoid gaps. The vessels originated from the facial arteries, and the most likely represented their submental branches (Figure 2c).
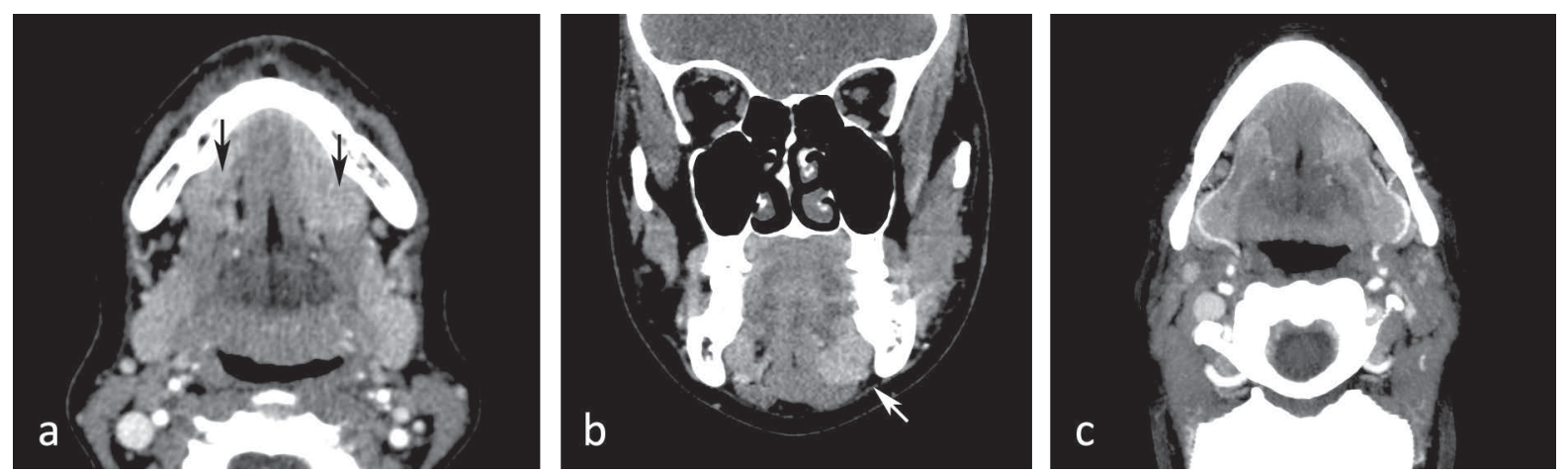

Figure 2. a) Axial contrast-enhanced CT image shows bilateral herniation of the sublingual glands through mylohyoid defect into the submandibular space (arrows). Note the enlargement of the left sublingual gland; b) Mylohyoid defect and sublingual gland herniation better depicted on the coronal reformatted CT image (arrows); c) Oblique axial MIP CT image revealed submandibular arteries passing through the mylohyoid defect adjacent to the sublingual glands

The patient was recalled for repeated US examination one week after CT. During US examination, the patient was asked to press the tongue down. The reversible herniation of the sublingual gland was then clearly depicted (Figure 3a). Tongue pressure-induced mylohyoid gap enlargement and herniation of the larger portion of the sublingual gland into the submandibular space. The blood vessel was also visualized traversing the mylohyoid gap (Figure 3b), which compression in the moment of a tongue pressure provoked subjective perception of pulsations. 

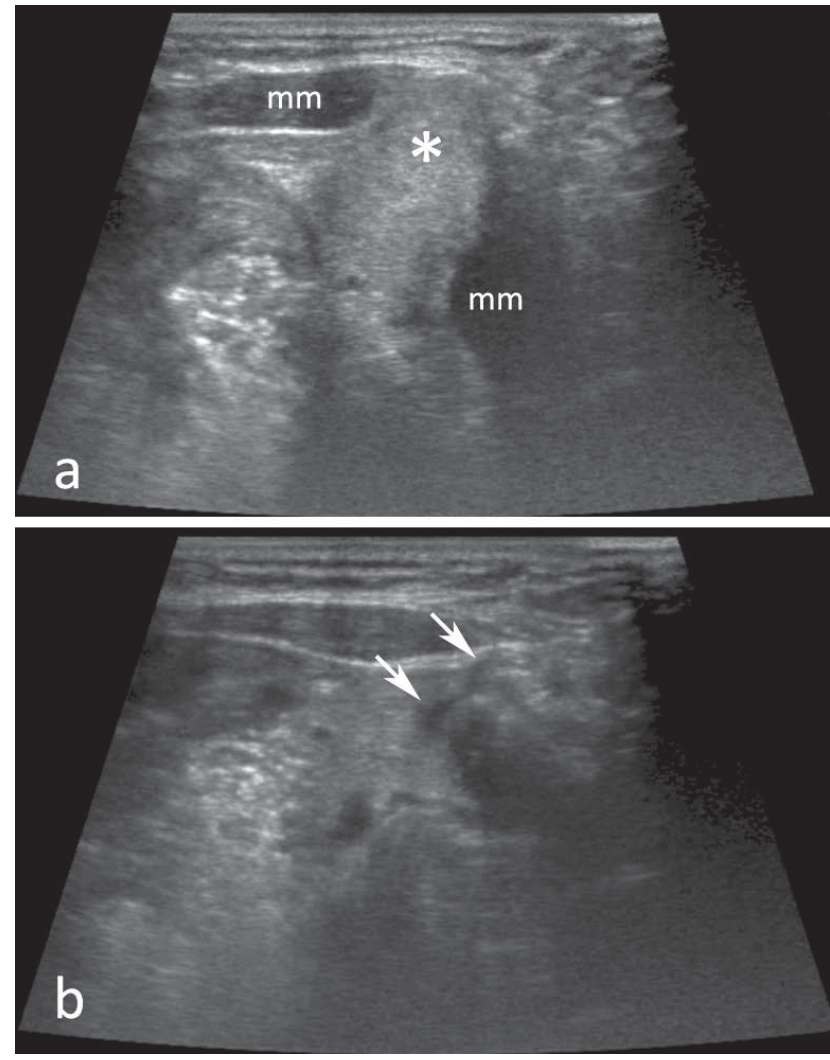

Figure 3. Axial US image of the left submandibular space during voluntary pressure by the tongue. a) Tongue pressure-induced larger herniation of the sublingual gland (asterisk) through the mylohyoid gap (mm-mylohyoid muscle); b) Note the blood vessel in the region of the mylohyoid defect (arrows)

\section{Case Report II}

The second patient was a 67-year-old non-smoker female who underwent a total thyroidectomy due to micropapillary carcinoma (follicular variant, stage T1aN0M0) six months before the onset of symptoms. During the routine follow-up visit, the patient complained of persistent mild to moderate pain in the left submandibular area. The pain had been presented for six weeks. There was a palpable moderately painful mass in the left submandibular region, around $2 \mathrm{~cm}$ in size. The overlying skin was normal in appearance. There were no other abnormalities on physical examination. Serum thyroglobulin level was $0.4 \mathrm{ng} / \mathrm{ml}$ so that the thyroid cancer metastasis was less probable.

On US examination, an oval soft-tissue mass was revealed in the left submandibular space (Figure 4a). The mass was hyperechoic and homogeneous in appearance. There were no visible changes in submandibular glands nor in the neck lymph nodes. No cystic or solid masses were seen in the thyroid gland region. A routine $\mathrm{CT}$ examination with intravenous contrast showed $4 \mathrm{~mm}$ - and $6 \mathrm{~mm}$-large defects in the anterior third of the right and left mylohyoid muscle, respectively. Defects contained fat tissue and blood vessels. The left sublingual gland was identified in the submandibular space protruding completely through the mylohyoid defect (Figure 4b,c). The right sublingual gland was smaller and located in its normal anatomical position. Both salivary glands showed homogeneous enhancement without focal changes in density. No other abnormalities were seen on $\mathrm{CT}$.
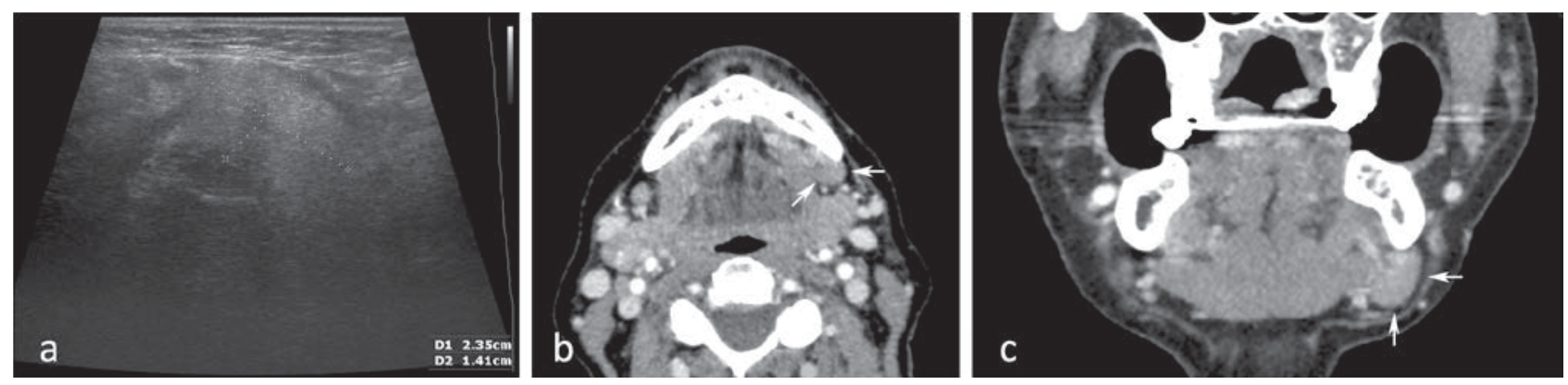

Figure 4. a) Axial US image shows an ovoid soft-tissue mass in the left submandibular space. b) On an axial contrast-enhanced CT image, the mass corresponds to the completely herniated sublingual glands through the mylohyoid gap (arrows). Note the blood vessels in the region of the gap. c) The appearance of the sublingual gland herniation on coronal reformatted CT image (arrows)

\section{Discussion}

The mylohyoid boutonnière is a common incidental finding during head and neck imaging ${ }^{6,8}$. Radiological studies have reported that unilateral or bilateral mylohyoid defects in $58 \%$ and $77 \%$ of asymptomatic patients during routine US and CT examinations, respectively ${ }^{6,8}$. Sublingual gland tissue may be radiologically detected in one-third of patients with mylohyoid boutonnière, while only $2 \%$ exhibit permanent herniation ${ }^{6,8}$. Kiesler et al. ${ }^{8}$ recently described three different types of relationships between mylohyoid boutonnière and sublingual gland on US. The type I was the most frequent and represented mylohyoid muscle thinning without sublingual gland herniation. Type II was described as a defect within the mylohyoid muscle with a sublingual gland herniation only during swallowing. Permanent herniation of the gland through the mylohyoid boutonnière (type III) was the least frequent. 
Despite the frequency of the mylohyoid boutonnière, symptomatic sublingual gland herniation had been only occasionally reported (Table 1). There was no age or gender predilection for the symptomatic herniation. The majority of the published cases presented with painless submandibular swelling, intermittent or permanent ${ }^{11-13}$. Only one patient had otalgia associated with sublingual gland herniation ${ }^{14}$, but the clinical/anatomical basis of such distant pain was not provided. The first patient presented in this paper also complained of moderate otalgia, while the second reported local submandibular pain. However, it is still not clear enough why sublingual gland herniation may manifest with pain. Histological analysis of cadaveric specimens showed signs of chronic inflammation in many sublingual glands herniating through the mylohyoid boutonnière ${ }^{5}$. Knowing that chronic inflammation induces a gradual decrease in salivary flow rate, this could predispose easier bacterial affection of the gland and consequent acute sialadenitis. In favor of this hypothesis is the fact that sublingual glands appeared enlarged on imaging in the majority of symptomatic cases.

Table 1. Review of cases of symptomatic sublingual gland herniation through the mylohyoid boutonnière published up to date

\begin{tabular}{lcccll}
\hline Author & No of cases & Gender & Age & Symptoms & Imaging modality \\
\hline Hopp et al., 2004 & 1 & M & 14 & Swelling during swallowing & US, CT, MR \\
Keberle et al., 2005 & 2 & M & 18 & Swelling during swallowing & MR \\
Ahmed et al., 2009 & 1 & M & 48 & Persistent swelling & MR \\
Sher \& Tan, 2016 & 1 & M & 74 & Swelling & Core throat, \\
& & & & Left-sided otalgia & MR \\
Current study & 2 & F & 34 & $\begin{array}{l}\text { Painful swelling and pulsations } \\
\text { Left-sided otalgia }\end{array}$ & US, CT \\
& & F & 67 & Submandibular pain & US, CT
\end{tabular}

The relationship between otalgia and sublingual gland herniation is more intriguing. Anatomical studies did not report any nerves or their branches passing through the mylohyoid boutonnière. In general, the ear has a complex sensory innervation that originates from trigeminal, facial, glossopharyngeal, vagus, and the great auricular nerve ${ }^{1}$. Theoretically, this means that any pathological condition involving the tissues and organs innervated by the abovementioned nerves or their branches may secondarily manifest with otalgia. In the case of sublingual gland herniation, the most probable route of the pain irradiation to the ear is via a lingual nerve. This nerve is the branch of the mandibular division of the trigeminal nerve, which gives sensory branches to the ear ${ }^{1}$. The curse of the lingual nerve is close to the mandibular body, submandibular salivary gland, and mylohyoid muscle, giving the sensory branches for the mucosa of the mouth floor. It is also connected with the facial nerve by chorda tympani, which passes through the middle ear ${ }^{1}$.

According to the cadaveric studies, communicating blood vessels between the submental and sublingual artery is a common content of the mylohyoid boutonnière ${ }^{5,7,10}$. On CT examination, blood vessels passing through the mylohyoid boutonnière may be detected in $35 \%$ of asymptomatic patients ${ }^{6}$. The presence of the blood vessels penetrating the mylohyoid muscle without detectable defect were also described on CT 6 but without any symptom. To the best of our knowledge, the patient presented in this paper is the first to complain of intermittent pulsation associated with the mylohyoid boutonnière. As confirmed by US, pulsations were provoked by the tongue pressure to the mouth floor and subsequent blood vessel compression by the herniated sublingual gland. The similar may happen during mouth opening and swallowing when contraction of the mouth floor muscles and increased intraoral pressure induces reversible herniation of the sublingual gland and blood vessel compression.

The knowledge of the sublingual gland herniation through the mylohyoid boutonnière and its clinical manifestations is of great importance because it may mimic other pathological entities both clinically and radiologically. Differential diagnosis in cases with submandibular swelling and pain most commonly includes salivary gland diseases (inflammation, stones, tumors), lymph node enlargement (either inflammatory or neoplastic), and developmental neck cysts. Beside clinical findings, radiological examination, especially ultrasonography, has an integral role in differentiating these entities. Salivary gland diseases (inflammation and stones) are accompanied with characteristic symptoms and signs. They may be clearly differentiated from sublingual gland herniation by US. In cases with 
suspected lymph node enlargement, US may differentiate inflammatory from the neoplastic origin with a great accuracy. Plunging ranula may share similar clinical and ultrasound appearance with intermittent sublingual gland herniation $^{15}$, like in the first case described in this paper. The mylohyoid defect is present in both conditions. However, ranula is typically filled with anechoic and homogenous fluid, whereas salivary gland tissue appears solid on US. Ranula often appeared in younger patients, while the sublingual gland herniation may be diagnosed at any age. The tongue induced compression to the floor of the mouth during US examination may be helpful in cases with intermittent herniation. If the sublingual gland herniation is persistent, US may not be enough, and the patient may need CT examination.

\section{Conclusions}

This is the first case of a mylohyoid boutonnière related intermittent vascular compression. Dynamic US and CT may help to differentiate intermittent and persistent sublingual gland herniation through the mylohyoid boutonnière from other pathological lesions in the submandibular region.

\section{References}

1. Standring S. Gray's anatomy: the anatomical basis of clinical practice. Edinburgh: Churchill Livingstone/Elsevier; 2008.

2. Forget M. De la coexistence de la grenouillette sublinguale et d'un kyste sus-hyoidien difficulté du diagnostic traitement. Bull Soc Chir, 1870,11:306.

3. Delens E. De la grenouillette sus-hyoidienne. Rev Chir, 1881;1:209-220.

4. Nathan H, Luchansky E. Sublingual gland herniation through the mylohyoid muscle. Oral Surg Oral Med Oral Pathol, 1989;59:21-23.

5. Engel JD, Stanton DH, Cohen DM, Lincoln N. Mylohyoid herniation: gross and histologic evaluation with clinical correlation. Oral Surg, 1987;63:55-59.

6. White DK, Davidson HC, Harnsberger HR, Haller J, Kamya A. Accessory salivary gland tissue in the mylohyoid boutonniere: a clinical and radiologic pseudolesion of the oral cavity. Am J Neuroradiol, 2001;22:406-412.
7. Windisch G, Weiglein A, Kiesler K. Herniation of the mylohyoid muscle. Craniofac Surg, 2004;15:566-569.

8. Kiesler K, Gugatschka M, Friedrich G. Incidence and clinical relevance of herniation of the mylohyoid muscle with penetration of the sublingual gland. Eur Arch Otorhinolaryngol, 2007;264:1071-1074.

9. Otonari-Yamamoto M, Nakajima K, Tsuji Y, Curtin HD, Hanyuda H, Okano T, et al. Mylohyoid muscle defects: comparison of CT findings and dissected specimens. Oral Radiol, 2011;27:50-56.

10. Yang HC, Kim SY, Kim SK, Oh CS, Chung IH, Nam KI. A cadaveric study on mylohyoid herniation of the sublingual gland. Eur Arch Otorhinolaryngol, 2016;273:4413-4416.

11. Hopp E, Mortensen B, Kolbenstvedt A. Mylohyoid herniation of the sublingual gland diagnosed by magnetic resonance imaging. Dentomaxillofac Radiol, 2004;33:351-353.

12. Keberle M, Eulert S, Relic A, Hahn D. Functional MR imaging of submandibular herniation of sublingual tissues through a gap of the mylohyoid muscle in two cases of submandibular "masses". Eur Radiol, 2005;15:1326-1328.

13. Ahmed M, Strauss M, Kassaie A, Shotelersuk V, DeGuzman R. Bilateral submandibular gland aplasia with clinicoradiological mass due to prolapsing sublingual salivary tissue through mylohyoid boutonnière: a case report and review. Dentomaxillofac Radiol, 2009;38:121-124.

14. Sher ZA, Tan G. Unilateral sublingual salivary gland hypertrophy with herniation through a boutonnière defect and contralateral sublingual gland hypoplasia. BJR Case Rep, 2016;2:20150382.

15. Jain P, Jain R, Morton RP, Ahmad Z. Plunging ranulas: high-resolution ultrasound for diagnosis and surgical management. Eur Radiol, 2010;20:1442-1449.

Conflict of Interests: Nothing to declare.

Financial Disclosure Statement: Nothing to declare.

Human Rights Statement: All the procedures on humans were conducted in accordance with the Helsinki Declaration of 1975, as revised 2000. Consent was obtained from the patient/s and approved for the current study by national ethical committee.

Animal Rights Statement: None required.

Received on March 3, 2021.

Revised on April 28, 2021.

Accepted on Jun 19, 2021.

Correspondence:

Aleksa Janović

Department of Diagnostic Radiology

Faculty of Dental Medicine, University of Belgrade, Serbia

e-mail: aleksa.janovic@stomf.bg.ac.rs 